A N N A L E S Annales de Bretagne et des Pays de l'Ouest

Les espaces du catholicisme français contemporain. Territoires et identités communautaires en tension

\title{
Samuel Gicquel
}

\section{(2) OpenEdition}

1 Journals

\section{Édition électronique}

URL : https://journals.openedition.org/abpo/7168

DOI : $10.4000 / a b p o .7168$

ISSN : 2108-6443

Éditeur

Presses universitaires de Rennes

\section{Édition imprimée}

Date de publication : 29 septembre 2021

Pagination : 228-230

ISSN : 0399-0826

\section{Référence électronique}

Samuel Gicquel, «Les espaces du catholicisme français contemporain. Territoires et identités

communautaires en tension ", Annales de Bretagne et des Pays de l'Ouest [En ligne], 128-3 | 2021, mis en ligne le 29 septembre 2021, consulté le 11 octobre 2022. URL : http://journals.openedition.org/abpo/ 7168 ; DOI : https://doi.org/10.4000/abpo.7168 
contributions réunies dans cet ouvrage s'attachent à restituer au mieux ces vestiges sous l'angle à la fois technique, stylistique et iconographique. C'est ainsi tout un monde polychrome de scènes lacunaires, de corps et de visages plus ou moins fantomatiques qui se révèlent au regard contemporain : on citera notamment la très belle silhouette du pauvre (fin XIII -début XIV e siècle) auquel saint Martin (en grande partie disparu) donne la moitié de son manteau sur les murs de la chapelle castrale de Châteaugiron (G. Fray et D. Jugan); le superbe concert des anges musiciens peints sur les lambris de la chapelle Notre-Dame-de-Carmès en Neulliac de la fin du XV ${ }^{e}$ siècle (P. L. Constantin) ou encore les dix saints en pieds du XVII ${ }^{\mathrm{e}}$ siècle de l'église du Quillio dans les Côtes-d'Armor (M. Hamoury). À défaut d'être exhaustif, mentionnons encore, pour le $\mathrm{xx}^{\mathrm{e}}$ siècle, l'étude de G. Kazerouni consacrée à la frise du Travail, œuvre de Camille Godet (1879-1966) pour la décoration de la Maison du Peuple à Rennes.

Si résurrection il y a, celle-ci n'est cependant jamais intégrale et les interventions restauratrices prennent garde désormais à ne pas se dissimuler tout en visant la réversibilité (voir à cet égard la restauration exemplaire du cycle de la vie de sainte Anne à l'église du Guerno par G. Fray et I. Hans-Collas). L'importance des lacunes dans la restitution des peintures antérieures au XIX ${ }^{\mathrm{e}}$ siècle laisse encore souvent une marge d'incertitude pour l'identification iconographique de certaines scènes. D'autres, quoique bien répertoriées, ne manqueront pas d'attirer l'attention (comme celle de cette femme agenouillée devant saint Mériadec coiffée d'une cloche miraculeuse dans l'église de Stival à Pontivy, p. 194). La riche et souvent inédite iconographie en couleur qui multiplie les aperçus d'ensemble ou de détail sur les décors étudiés se révèle de ce point de vue très pertinente, tout en donnant à l'objet le statut de " beau livre ".

L'ouvrage montre bien également que la question de la préservation de ces peintures n'est pas seulement d'ordre technique : elle procède aussi de l'évolution du regard inévitablement culturel que les sociétés posent sur elles et la notion actuelle très prégnante de patrimoine rencontre parfois ses limites en raison du statut même de l'art en question. Ainsi, une réalisation typique du street art, quand bien même celle-ci résulterait d'une commande officielle et porterait la "signature " d'un artiste de renommée internationale, a-t-elle vocation à être préservée? (C. Vautier). La patrimonialisation pose également des problèmes d'ordre politique et mémoriel : faut-il, par exemple, protéger les dessins et autres graffitis plus ou moins aboutis laissés par les soldats d'Hitler dans les bunkers du Mur de l'Atlantique? (C. Perrichot). On le voit, cet ouvrage ne s'interdit aucune question et embrasse très largement le thème des peintures monumentales en Bretagne, contribuant ainsi à renouveler en profondeur le regard porté sur ces images rescapées d'un monde disparu ou même - si l'on songe aux graffitis - en voie de disparaître sous nos yeux.

Franck Mercier

HERBINET, Vincent, Les espaces du catholicisme français contemporain. Territoires et identités communautaires en tension, Presses universitaires de Rennes, Rennes, 2021, 329 p.

L'ouvrage de Vincent Herbinet est le produit de sa thèse de doctorat en histoire, soutenue à l'université de Lyon 2 en 2018 et consacrée aux récentes mutations du catholicisme français. Son objectif n'était pas tant d'analyser les décompositions de la vieille chrétienté que de mettre en lumière les recompositions observées à partir des années 1980, c'est-à-dire essentiellement sous les pontificats de Jean-Paul II et de Benoît XVI, le travail se refermant en 2016. L'étude est une approche comparée 
de trois diocèses, qui constituent des observatoires du catholicisme contemporain : le diocèse de Rennes, dont l'auteur est originaire, et qui offre une fenêtre sur la christianitude bretonne; le diocèse d'Autun, caractérisé par la ruralité et la présence du sanctuaire de Paray-le-Monial; et le diocèse de Fréjus-Toulon, dont la gouvernance est fortement marquée par la personnalité de $\mathrm{M}^{\mathrm{gr}} \mathrm{Rey}$, son évêque " tradismatique ", c'est-à-dire issu de la sphère traditionaliste et charismatique. On le devine à travers les choix opérés : l'un des enjeux de l'étude est de réfléchir à la place des communautés nouvelles. Géographe de formation, Vincent Herbinet accorde une place centrale à l'espace et livre un travail hybride, qui emprunte à la fois à l'histoire, à la géographie et à la sociologie. C'est une des richesses de la thèse, mais, simultanément, peut-être aussi une de ses faiblesses, en particulier pour les amateurs d'histoire, qui pourront regretter l'absence d'un séquençage chronologique plus visible, notamment au niveau du plan.

L'ouvrage se divise en trois parties thématiques. La première est décrite par l'auteur comme une analyse " de la relation du catholicisme à la postmodernité ". En clair, il s'agit de faire le point sur les adaptations face à la crise du catholicisme. Vincent Herbinet dresse un bilan des mutations paroissiales des dernières années et décrit avec précision l'émergence de pôles pastoraux dans les diocèses de Rennes et d'Autun. Le diocèse de Fréjus-Toulon, qui fait pourtant partie du cadre d'études, est lui curieusement oublié. L'auteur consacre ensuite d'amples développements à quelques dossiers emblématiques - l'Action catholique ou les Associations familiales catholiques par exemple - pour évoquer les dynamiques militantes et mettre en lumière l'émergence de mouvements identitaires qui cherchent à redonner de la visibilité au catholicisme dans un monde sécularisé. Dans cette première partie, on retrouve donc les observations de Louisa Plouchart et de Yann Raison du Cleuziou, qui ont analysé ces évolutions, l'une en enquêtant sur les mutations paroissiales dans le diocèse de Rennes, l'autre en étudiant les catholiques observants. Elle s'imposait pour planter le décor.

La mise en valeur de logiques polarisantes et communautaires amène logiquement Vincent Herbinet à se pencher dans une seconde partie sur l'unité des diocèses étudiés et sur la façon dont ils sont gouvernés. Au fil des pages, apparaissent nettement les différents modes de gouvernance ecclésiale, portés par des figures aux sensibilités diverses. Dans le diocèse de Rennes, l'épiscopat de $\mathrm{M}^{\mathrm{gr}}$ d'Ornellas se caractérise par la mise en place, à partir de 2009, des "pôles eucharistiques rayonnants ", qui fédèrent une communauté autour d'un bourg ou d'une ville dynamique. Selon $\mathrm{M}^{\mathrm{gr}}$ d'Ornellas, qui établit une analogie avec le système cardiaque, ce pôle doit fonctionner dans un mouvement de diastole-systole, avec une impulsion du centre, qui doit se nourrir de ce que renvoient les périphéries. Parallèlement, la participation des laïcs a été encouragée, notamment à travers la démarche synodale. Le diocèse de Fréjus-Toulon se caractérise pour sa part par un pouvoir épiscopal fort et l'imposition par le haut d'une ligne pastorale qui promeut la liturgie traditionnelle et encourage l'installation des communautés nouvelles. L'auteur n'ignore pas les oppositions que cette stratégie suscite, en interne comme en externe, mais on aurait aimé qu'il les analyse davantage, quitte à passer plus vite sur l'histoire de la communauté Saint-Martin, certes intéressante, mais largement extérieure aux diocèses étudiés.

La troisième partie, consacrée au " catholicisme attestataire ", est celle qui apporte le plus de nouveautés. Elle présente avec précision la genèse des éléments qui composent la galaxie traditionaliste, en accordant une large place à l'émergence du Renouveau charismatique. Dans le diocèse de Rennes, l'installation du groupe de prière Notre-Dame de Joie, soutenue par le clergé local, et celle de l'Emmanuel, issue d'une greffe extérieure, sont par exemple exposées en détail par Vincent 
Herbinet, qui parvient à retracer l'histoire de ces groupes grâce à la collecte de nombreux témoignages. L'ouvrage montre un catholicisme créatif, en quête de visibilité, polarisé autour de places fortes - la paroisse Saint-Hélier à Rennes, le sanctuaire de Paray-le-Monial dans le diocèse d'Autun. Il n'ignore pas les tensions qui en résultent, aussi bien dans le clergé que parmi les fidèles, à l'exemple de celles qui secouèrent la paroisse de Dinard en 2012-2013, quand des paroissiens s'inquiétèrent du projet d'installation de l'Emmanuel (p. 238). Les démonstrations sont étayées et, à la lecture de cette partie, on se demande si l'auteur n'aurait pas gagné à faire de ces communautés nouvelles, dont il est familier, un fil conducteur plus affirmé, voire à les mettre au cœur de son sujet.

Il a choisi une approche plus géographique. La démarche se défend, bien entendu, mais on peut regretter que rien ne soit dit sur l'usage du numérique qui participe à la déterritorialisation religieuse. C'est d'autant plus regrettable que les groupes charismatiques et traditionalistes dépeints dans l'ouvrage sont très actifs sur l'internet. En dépit de ce manque, la lecture de cet ouvrage s'avère fort utile pour comprendre les phénomènes de polarisation à l'œuvre dans le catholicisme contemporain. Polarisation : c'est sans doute le maître-mot de cet ouvrage, et la quintessence de ce qu'il faut en retenir. Elle est à la fois imposée par la crise des vocations et encouragée pour constituer de puissants môles de résistance voire de reconquête. Cette polarisation est simultanément territoriale, du fait des réformes paroissiales, et communautaire, en raison du développement d'un catholicisme affinitaire. Elle peut donc fragiliser l'homogénéité du tissu ecclésial. Vincent Herbinet parle avec raison des logiques de " marketing territorial " (p. 313) qui ont supplanté les logiques de proximité. À la lumière de son ouvrage, on pourrait aussi parler de marketing diocésain, comme le montre l'exemple du diocèse de Toulon, dont l'identité est aujourd'hui marquée par les communautés nouvelles et traditionalistes qui s'y sont installées.

Samuel Gicquel

VillaRes, Ramón, Botrel, Jean-François et Rivalan-GuéGo, Christine (dir.), Galicia-Bretaña. Olladas comparadas, Santiago de Compostela, Consello da Cultura Galega, 2020, 349 p. Traduction et révision des textes de Cristina Río López.

Cet ouvrage, publié par le Conseil de la Culture Galicienne et rédigé intégralement en langue galicienne, est l'un des fruits de la collaboration instaurée à partir des années 1970 et 1980 entre les universités de Saint-Jacques de Compostelle et de Rennes, auxquelles appartient la quasi-totalité des auteurs.

L'ouvrage s'ouvre sur un article de Jean-François Botrel retraçant la « découverte " de la Galice par les Bretons à partir du milieu des années 1960, intérêt concrétisé par la suite par des échanges culturels et des recherches universitaires sur certains aspects de la Galice. En miroir, Ramón Villares, dont la somme sur l'histoire de la Galice a été opportunément éditée en français par les Presses Universitaires de Rennes en 2019, évoque "les relations et les analogies entre [ces] deux nations culturelles ", depuis Moyen-Age mais essentiellement au cours des cent dernières années. C'est en effet à partir des années 1920, avec la Génération Nós (Nous) et la revue du même nom, que le galicianisme culturel regarde vers la Bretagne et y décèle une parenté avec la Galice. C'est, par exemple, lors d'un séjour en Bretagne qu'Alfonso Rodríguez Castelao découvre en 1928 les similitudes entre calvaires galiciens et bretons, consacrant aussitôt à ces derniers un ouvrage illustré par lui-même (As cruces de pedra na Bretaña, Saint-Jacques de Compostelle, 1930, réédition facsimilé, Galaxia, Vigo, 1992). 